\title{
Active Flutter Suppression Controllers Derived from Linear and Nonlinear Aerodynamics: Application to a Transport Aircraft Model
}

\author{
Josiah M. Waite, ${ }^{*}$ Bret K. Stanford, ${ }^{\dagger}$ Robert E. Bartels, ${ }^{\ddagger}$ Walter A. Silva, ${ }^{\S}$ and Steven J. Massey ${ }^{\dagger}$ \\ NASA Langley Research Center, Hampton, VA, 23681
}

Active flutter suppression has been demonstrated in simulation by many researchers, generally using methods based on linear aerodynamics and often with simplistic geometries. In this paper, active flutter suppression is demonstrated in a simulation using a Navier-Stokes aerodynamics code, FUN3D, and a realistic transport aircraft configuration. This is accomplished using simple observer-feedback controllers derived from linear aeroelastic models, including reduced order models built via FUN3D data. The development of these reduced order models is described here. It is shown that controllers derived from reduced order models of the nonlinear aerodynamics outperform controllers based on linear aerodynamics.

\section{Nomenclature}

$\begin{array}{ll}\text { AFS } & =\text { Active flutter suppression } \\ \text { ASE } & =\text { Aeroservoelastic } \\ \mathrm{CRM} & =\text { Common research model } \\ \mathrm{DLM} & =\text { Doublet lattice method } \\ \mathrm{FFT} & =\text { Fast Fourier transform } \\ \mathrm{GAF} & =\text { Generalized aerodynamic force } \\ \mathrm{LQR} & =\text { Linear quadratic regulator } \\ \mathrm{LQG} & =\text { Linear quadratic Gaussian } \\ \mathrm{RANS} & =\text { Reynolds-averaged Navier Stokes } \\ \mathrm{ROM} & =\text { Reduced order model } \\ \mathrm{A}, \mathrm{B} & =\text { Estimator state matrices } \\ b & =\text { Wingspan } \\ d t & =\text { Time step size (seconds) } \\ \mathrm{H} & =\text { Sensor matrix } \\ J & =\text { Control cost } \\ \mathrm{K}, \mathrm{L} & =\text { Feedback and Kalman gain matrices } \\ \mathrm{Q}, \mathrm{R} & =\text { State \& control weighting matrices } \\ q & =\text { Dynamic pressure (kPa) } \\ u & =\text { Input control surface amplitude vector } \\ V_{D} & =\text { Dive speed } \\ \hat{x} & =\text { Estimator state vector } \\ y & =\text { Wing spanwise coordinates } \\ \ddot{\delta} & =\text { Physical accelerations } \\ \ddot{\eta} & =\text { Modal accelerations } \\ \lambda & =\text { Measure of decay of system } \\ & \end{array}$

\footnotetext{
* Research Aerospace Engineer, Aeroelasticity Branch, MS 340, josiah.m.waite@ nasa.gov

${ }^{\dagger}$ Research Aerospace Engineer, Aeroelasticity Branch, MS 340, AIAA Senior Member

$\dashv$ Senior Research Engineer, Aeroelasticity Branch, MS 340, AIAA Senior Member

${ }^{\S}$ Senior Research Scientist, Aeroelasticity Branch, MS 340, AIAA Associate Fellow
} 


\section{Introduction}

$\mathrm{T}$ HOUGH not commonly used on current fixed-wing aircraft due to the inherently high risk, active flutter suppression (AFS) systems have been studied in a research context for many years, as summarized in the recent review article by Livne[1]. AFS involves the use of active control surfaces to artificially expand the flutter instability boundary of a flexible wing via closed-loop control. The reduced threat of flutter-based failures could allow for lighter-weight aircraft structures during the design process (which could potentially lead to significant fuel cost savings), though this is only true if one can guarantee that the control surfaces will always be properly operational. Such a risk has historically limited the use of AFS on civilian aircraft, though sustained research in this field will hopefully lead to more robust systems and greater utilization in civilian and commercial applications. Many studies conducted to this point in the area of AFS have been experimental in nature [2-5]. When numerical studies of flutter suppression have been conducted, they have traditionally been performed within the framework of an aeroservoelastic (ASE) model that includes computational models for the structure, the fluid, and some control law. Typically, a linear formulation has been used for the fluid solver to keep the computational cost of the analysis down [4, 6-9]. The reduction in computational cost, however, often comes at the price of reduced accuracy in predicting the fluid dynamics, particularly for transonic flows. For example, consider Fig. 11, which shows the predicted flutter envelope as calculated via a linear doublet lattice method (DLM) and a nonlinear Navier-Stokes solver, FUN3D[10] (Fully Unstructured Navier-Stokes 3D). The results from the linear and nonlinear analyses have similar trends in the subsonic regime, but they diverge significantly in the transonic regime near Mach 0.9. The linear code is unaware of the nonlinear transonic "flutter dip" that is predicted by FUN3D (and that physically occurs in experiments). This is likely due to a region of mixed flow over the wing (a supersonic bubble), which oscillates in response to wing deformations. Since modeling aeroelastic phenomena in the transonic flight regime is of interest to many researchers, this inability to capture transonic aerodynamic nonlinearities limits the problems to which the ASE model can be applied.

Furthermore, as aircraft designs tend toward wings with higher aspect ratio and increasing flexibility, interactions between structural dynamics and aerodynamics become more complex and the ability to generate accurate, nonlinear aerodynamic predictions becomes increasingly essential to aircraft design[11]. Although researchers have successfully integrated higher-order CFD solvers into computational aeroelastic models (Refs. 12, 13), it is still considered to be a challenge because of the high computational cost[14], particularly for unsteady simulations. Because of this, there are only a handful of dynamic aeroelastic analyses done using higher-order CFD solvers, and most of those are done using simple geometries. There are even fewer ASE models that integrate CFD solvers, and still fewer[15-17] that use realistic aircraft geometries.

The goal of this work, therefore, is to demonstrate active flutter suppression within a computational ASE model that uses a nonlinear aerodynamics solver on a realistic airplane configuration. The nonlinear aerodynamics solver

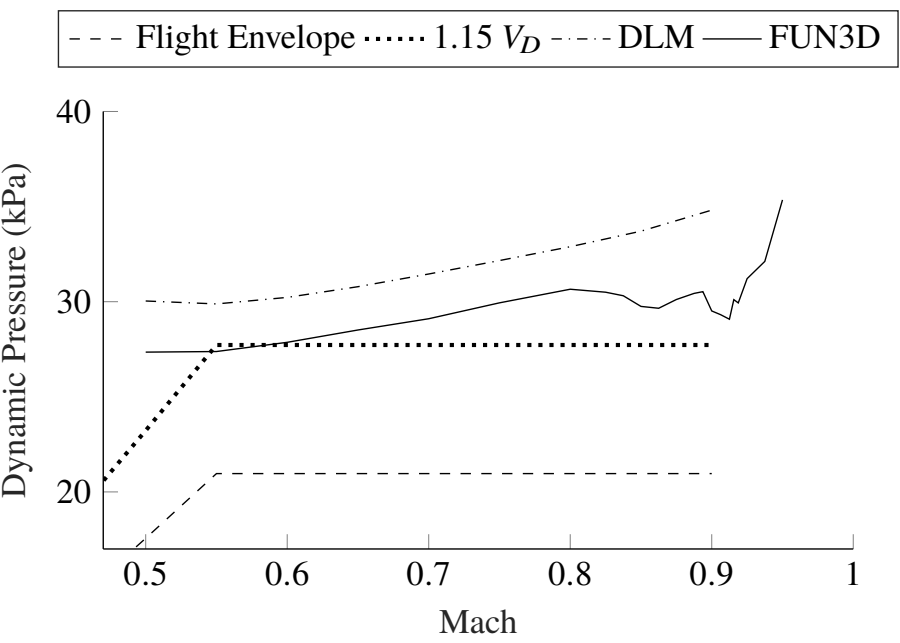

Fig. 1 Flutter envelope for the NASA Common Research Model as computed via a linear doublet lattice method (DLM) and a nonlinear Navier-Stokes solver, FUN3D. The other two curves represent the flight envelope and the minimum required aeroelastic stability margin, which is $15 \%$ higher than an airplane's design dive speed, $V_{D}$. 
and the linear structural dynamics solver from the NASA Langley FUN3D code will be used, and all AFS studies will be demonstrated within that framework. The controllers and estimators used for AFS, however, cannot be directly generated from FUN3D, owing to the extremely large size of the numerical systems. Instead, more tractable numerical models are used to generate the controllers, of which two are used. One is derived from a linear aeroelastic model, with DLM aerodynamics converted into state-space form via the typical Roger approximation. The other is derived from a reduced-order model (ROM) of the nonlinear fluid dynamics as computed by FUN3D. An additional goal of this work is to show that a controller derived from a linear aerodynamics code (like DLM) will not be as effective at controlling an unstable system as a controller created using a nonlinear aerodynamics-based ROM, due to the importance of transonic aerodynamics. Finally, the airplane geometry is based on the NASA Common Research Model (CRM), a generic transport configuration suitable for aeroelastic analysis, as developed in Ref. 18 .

\section{Methods}

The general workflow followed in this study was: (1) compute a linear state-space model of the flow, (2) design a linear controller and an estimator, and (3) tie the controller, the estimator, and FUN3D together to form an ASE framework where AFS can be demonstrated.

\section{A. Geometry}

The analyses presented here were conducted on a half-span model of the NASA CRM (see top left image in Fig. 2) as developed in Ref. 18. This is a generic transport geometry with a cruise Mach number of 0.85, wing span of 58.7 meters, mean aerodynamic chord of 7 meters, aspect ratio of 9 , taper ratio of 0.275 , and sweep angle of $35^{\circ}$. Results presented in this paper are at Mach number of 0.85 and angle of attack of $0^{\circ}$. Two trailing edge control surfaces were attached to the wing as shown in the bottom of Fig. 2. When deflected, the edges of each trailing edge control surface were blended into the the edges of adjacent control surfaces, as shown in the top right of Fig. 2.

Aeroelastic analyses were accomplished by combining the aerodynamic solver within FUN3D with a modal structural dynamics solver, also within FUN3D. Only the wing was considered to be flexible in this work, populated with an aluminum wingbox built from ribs, spars, skins, and stiffeners, described more fully in Ref. 18. The wingbox was connected to a series of lumped masses meant to emulate fuel and engine inertia. A total of 25 flexible structural modes and two rigid control modes (where the control surfaces were assumed to be irreversible) were generated for this geometry. The control surfaces associated with the two control modes were located at $2 y / b=0.214$ and 0.787 . Modes $1,2,3$, and 8 most closely resembled pure bending and torsional modes, so they are the only modes for which results are presented. The final closed-loop FUN3D simulations used all 25 structural modes, while the FUN3D computations for

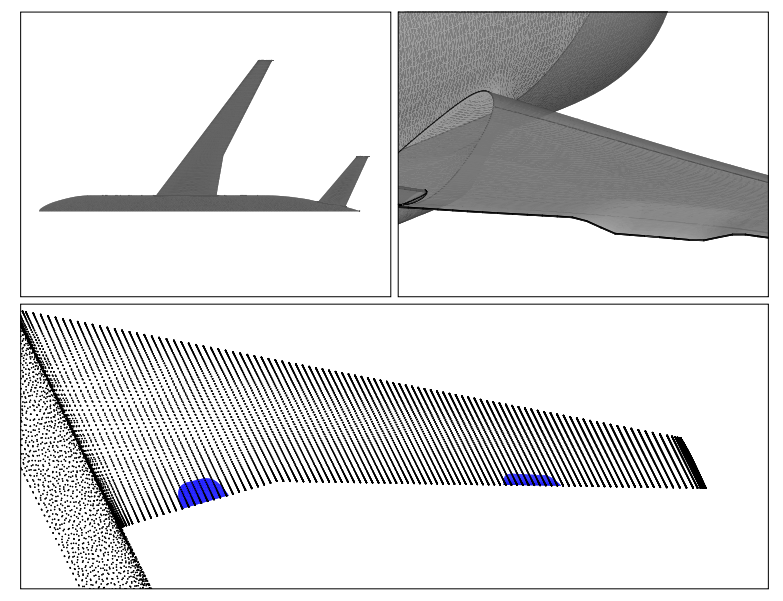

Fig. 2 Top left: Visualization of the NASA CRM half-span model used in the current analysis. Top right: Illustration of one control surface deflection. Note the control surface blending. Bottom: Representation of the two control surfaces used in the current ASE model, with control surface \#1 being closest to the root and control surface \#2 being closest to the tip. 
the ROMs used less. Figure 3 shows (qualitatively) the vertical deflection magnitudes for modes 1, 2, 3, and 8, which had natural frequencies of $10.7,23.6,26.4$, and $85.0 \mathrm{rad} / \mathrm{s}$, respectively.

\section{B. FUN3D}

NASA Langley's FUN3D code was used for both the computational fluid dynamics and structural dynamics analyses. While FUN3D allows a user to couple a fluid-structure interaction simulation using an external finite element code, it also has a built-in modal structural dynamics equation solver that uses a second-order accurate predictor-corrector scheme [19]. This built-in linear structural dynamics solver was used here, and it was within the structural loop in the FUN3D code that closed-loop control was implemented.

Before performing any aeroelastic analysis (static or dynamic) in FUN3D, a converged steady flow simulation with a rigid aircraft configuration was obtained. An unsteady static aeroelastic simulation was then restarted from the converged steady solution. Once the unsteady static simulation reached a converged state, a disturbance was introduced into the system and an unsteady dynamic aeroelastic simulation was restarted from the static aeroelastic solution. For flutter analysis, a typical disturbance is a pulse to one of the flexible mode velocities. For the dynamic aeroelastic simulations used to develop the ROMs in this work, the disturbances were the commanded control surface motions. For all FUN3D analyses, the unsteady Reynolds-averaged Navier-Stokes (RANS) equations were solved using a second-order upwind scheme with a Spalart-Allmaras turbulence model. A fairly coarse semi-infinite tetrahedral volume mesh was used here, with 3.1 million nodes, and a y+ value nearly 1 throughout the surface. For the closed-loop dynamic aeroelastic analyses, the time step size varied between $2.9 \mathrm{E}-3$ and $3.3 \mathrm{E}-3 \mathrm{~s}$, and each time step included 20 fluid subiterations.

\section{Linear Models used to Generate Estimators \& Controllers}

Three linear state-space models were developed from which controllers could be derived. The aerodynamics of the first were computed using a DLM. The second and third state-space models were data-driven ROMs that had been trained using nonlinear FUN3D solutions. Note that, while based on nonlinear solutions, the ROMs themselves were linear models. These ROMs were generated using different methods and will be referred to as ROM-G and ROM-W (so named based on their inputs: ROM-G used Gaussian pulses as inputs, while ROM-W used Walsh functions). The process used to develop each state-space model will be briefly discussed.

\section{DLM}

Well-established procedures (summarized in Ref. 20, for example) were used to derive a linear aeroservoelastic model built on the DLM. The first step was to compute the frequency domain aerodynamics by executing the DLM code. Next, structural mode shapes and control surface mode shapes were used to derive structure-on-structure and control-on-structure generalized aerodynamic forces. The well-known Roger approximation (Ref. 21) was then used to convert the frequency domain aerodynamics into the time domain via rational function approximations.
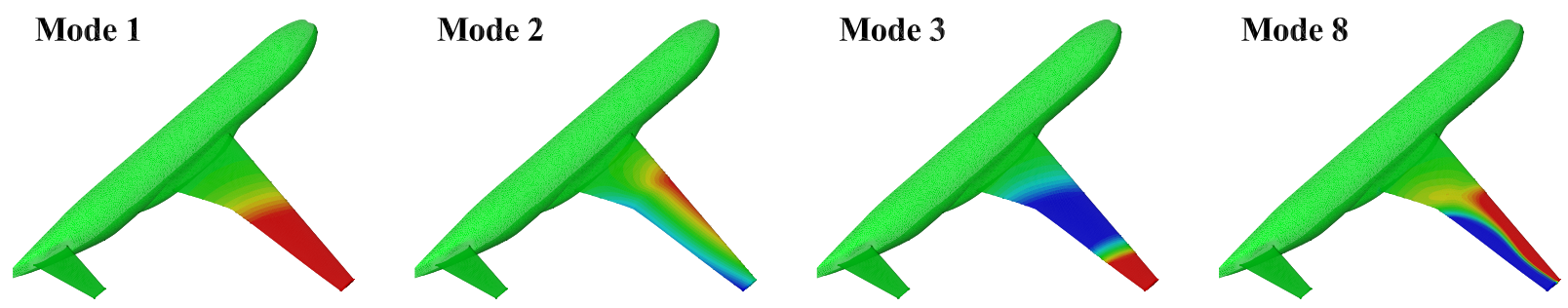

Fig. 3 Vertical deflections associated with modes 1, 2, 3, and 8. Although many more modes were generated for the analyses presented here, these modes most closely resembled pure bending and torsional modes, so they are the modes for which results are presented. 


\section{2. $R O M-G$}

The DLM, like any aerodynamic panel method, solves a simplified version of the full Navier-Stokes equations by making certain assumptions (e.g., irrotational flow or small perturbations). In this way, computations become quicker and cheaper. In contrast, the ROMs referred to here used FUN3D RANS simulations to "train" a linearized model. For the ROM-G, the process to obtain the state-space ROM can be found in Refs. 22 and 23 and is summarized here:

1) For each structural and control mode, do the following:

a) Excite the mode by introducing a Gaussian pulse to the modal displacement. Only one mode's displacement should be disturbed at a time, so all other modes' displacements are held constant at zero. Note that the Gaussian pulse should be shaped to excite a particular range of frequencies of interest.

b) With the Gaussian pulse in that mode's displacement as input, run a FUN3D simulation. This is the simulation that "trains" the ROM. Note that the length of the simulation and the time step size must be tailored to capture the desired frequency range: the overall length of the training simulation will set the lowest frequency that can be obtained, and the time step size will set the Nyquist frequency.

c) Compile the response of all modes due to the input.

2) Compile all responses due to all inputs.

3) Perform a fast Fourier tranform (FFT) on the inputs and outputs to get generalized aerodynamic forces (GAFs) in the frequency domain.

4) Apply the Roger approximation to get the data back into time domain.

5) Generate state-space matrices from time domain data.

ROM-G is a ROM for the unsteady aerodynamics only; aeroelastic coupling is not initially involved. The GAFs that result from this process can then be converted into the time domain, and then finally coupled with a structural model, where the dynamic pressure acts as a feedback gain.

\section{3. $R O M-W$}

AEROM[24], a suite of tools for generating aeroelastic ROMs, was used to obtain ROM-W. The general procedure was:

1) Excite all control modes simultaneously by introducing orthogonal Walsh functions to their modal displacements. Note that each Walsh function should be shaped to excite a particular range of frequencies of interest. Also note that the magnitudes of the input Walsh functions for this case were comparable to the magnitudes of the ROM-G inputs.

2) With the Walsh functions in the control modes' displacements as inputs, run a FUN3D simulation. As with ROM-G, this is the simulation that "trains" the ROM. Also, the same considerations regarding the length of the simulation and the time step size of ROM-G must be considered here.

3) Compile the responses of all the structural modes due to the inputs.

4) Exercise an algorithm within AEROM to get individual impulse responses of each mode due to individual control inputs.

5) Convert the impulse responses to state-space using an eigensystem realization algorithm, also included in AEROM.

In contrast to ROM-G, which is a ROM for the unsteady aerodynamics only, ROM-W is a ROM which does account for aeroelastic interactions from the beginning, and therefore is only valid at a single dynamic pressure (i.e., the pressure at which the ROM was trained). The AEROM tool does have the capability to use Walsh functions to train the unsteady aerodynamics only (and then couple to a structural model after the fact), and this capability will be explored for future work.

\section{Generation of the Control Law and the Estimator}

There has been much research done on nonlinear controllers and their application to aeroelastic systems (Refs. 25, 26), but the effectiveness of simpler, linear controllers was analyzed in this work. Specifically, it was desired to use simple full-state feedback controllers (linear quadratic regulator, LQR), where the aeroelastic state vector at a given time step would be multiplied by a feedback matrix, to produce the vector of control surface motions. Such an operation was not possible directly within FUN3D, as the number of states was extremely large (modal structural data, but also velocity, pressure, and density information at each node), and the requisite matrices were not easily accessible. Feedback control is only possible with smaller, more compact systems. Therefore, a small amount of data was taken from FUN3D at each time step ( 25 modal accelerations, $\ddot{\eta}$, converted to 21 vertical acceleration measurements along the wing, $\ddot{\delta}$ ), and used to 
estimate the full state vector of a stand-alone linear aeroelastic model. This linear aeroelastic state was then used to produce the commanded control surface rotations, which were fed to FUN3D. The development of these estimators and controllers followed the methods presented in Ref. 27, and is essentially a linear quadratic Gaussian (LQG) controller. The stand-alone linear aeroelastic estimator was integrated in time alongside FUN3D, and is assumed to be of the form:

$$
\dot{\hat{x}}=A \hat{x}+B u+L(\ddot{\delta}-H \hat{x})
$$

where $u$ is a $2 \times 1$ vector of control surface rotations and $\hat{x}$ is a vector of linear aeroelastic states, generated from one of the three linear models discussed in Section II.C, $\ddot{\delta}$ is the $21 \times 1$ vector of vertical accelerations along the wing, which comes from FUN3D. $H$ is a sensor coversion matrix: the term $H \hat{x}$ produces the 21 vertical accelerations as computed by the linear aeroelastic model. $L$ is a Kalman gain matrix whose derivation is given below. When $L$ is properly designed, the error between the FUN3D accelerations and the linear accelerations $(\ddot{\delta}-H \hat{x})$ will decay to zero over time.

Having obtained a compact aeroelastic state, feedback was assumed of the form:

$$
u=-K \hat{x}
$$

The feedback gain matrix (i.e., the controller), K, was found such that a cost function, $J$, was minimized:

$$
J=\frac{1}{2} \int_{0}^{\infty}\left(\|x\|_{Q}+\|u\|_{R}\right) d t
$$

where $\frac{1}{2} \int_{0}^{\infty}\|x\|_{Q} d t$ represents the energy of some combination of the states, and $\frac{1}{2} \int_{0}^{\infty}\|u\|_{R} d t$ represents the energy necessary for control; $Q$ is a state weighting matrix, which is a fixed quantity for each case; $R$ is a control weighting matrix. Larger values of $R$ relative to $Q$ penalize the controls more. The goal was to minimize the energy in the states without using too much energy in the control effort. The $L$ matrix from Equation 1 was generated using a similar equation to the $J$ cost function with $R$ and $Q$ being white noise inputs.

\section{E. ASE Model Overview}

Once the controllers were designed, they, along with the linear model from which they were derived, were implemented alongside FUN3D to create the ASE model, as shown in Fig. 4 . Note that, while Fig. 4 shows the controller and estimator as being separate from FUN3D, in actuality they were added into the FUN3D source code. They are shown separately here only for clarity. The ASE model's workflow was as follows:

1) Control modes are initially set to zero deflection.

2) Some initial disturbance is introduced into the system. In this case, an initial velocity was introduced to the first structural mode.

3) At each time step, FUN3D simulates the system's aeroelastic response to the input by calculating modal accelerations for each structural mode. These modal accelerations ( $\ddot{\eta}$ in Fig. 4) are the states of interest.

4) A simulated sensor, which is simply a matrix that converts the modal accelerations to physical accelerations at discrete points on the wing, represents accelerometer measurements as might be taken on a wind tunnel model. In this case, the sensor took the 25 modal accelerations and converted them to 21 accelerometer readings along the wing.

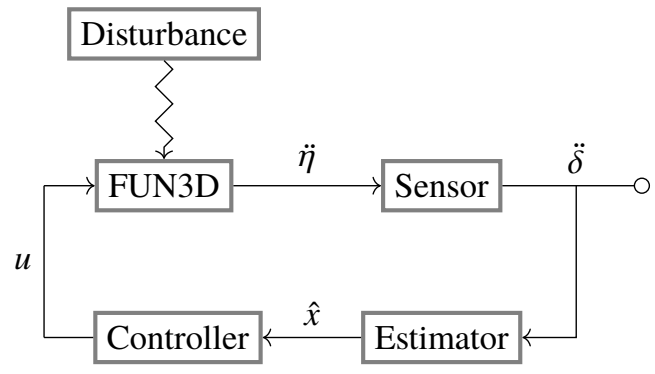

Fig. 4 Block diagram of the ASE model. Output from FUN3D is converted to 21 physical accelerations $(\ddot{\delta})$ by a sensor matrix. These accelerations are fed into an estimator, whose output goes to the controller. Two control surface commands $(u)$ are passed from the controller to FUN3D. 
5) The FUN3D accelerometer readings, $\ddot{\delta}$, are fed into the estimator at each time step. The estimator then uses this data to compute the estimate of the linear aeroelastic solution vector, $\hat{x}$.

6) The controller takes the estimated states and generates a set of commands to be applied to the control modes. These commands are passed to FUN3D, where the commands are processed and the control surfaces deflect to the commanded positions. The simulation advances to the next time step.

Note that there were two simulations marching through time together- FUN3D's unsteady RANS simulation and the estimator's linear simulation. Also note that this ASE framework uses a linear controller, derived from a linear model (though in the case of the ROMs, the linear model is itself trained from a RANS simulation), to control a nonlinear system.

\section{Results}

\section{A. Accuracy of State-Space Models}

In order to compare the linear codes to FUN3D (which is considered the "truth" model here), sinusoidal inputs of varying frequencies were applied to the modal displacement of control surface \#2 (see Fig. 2) and the open-loop responses were computed by each code. Figure 5 shows results from each of the linear codes compared to several results from FUN3D $(q \approx 17 k P a)$. Because the ROMs were intended to capture more of the nonlinear effects than the DLM, it was expected that the ROM-generated results would be closer to the higher-fidelity FUN3D results than the DLM-generated results. This, however, was not as apparent as expected. The DLM performed nearly as well as the ROMs for many frequencies, but there were a few notable instances where the ROMs appear to perform better: at low frequencies (0-12 rad/s) in mode 1, where the real parts from both ROMs predicted results closer to FUN3D; at frequencies in the 15-20 rad/s range in mode 2, where the real parts of ROM-G results were closer to FUN3D than both the DLM and ROM-W results and the imaginary parts of ROM-G and DLM were comparable; at high frequencies $(35-50 \mathrm{rad} / \mathrm{s})$ in mode 8 , where the real and imaginary parts of the ROMs' predictions matched FUN3D better than the DLM. It is also noted that, in the 25-30 rad/s range, there is no direct FUN3D data, and that range is where the DLM and the ROMs differ the most. It appears that, in those areas were the ROMs are performing better than the DLM, the

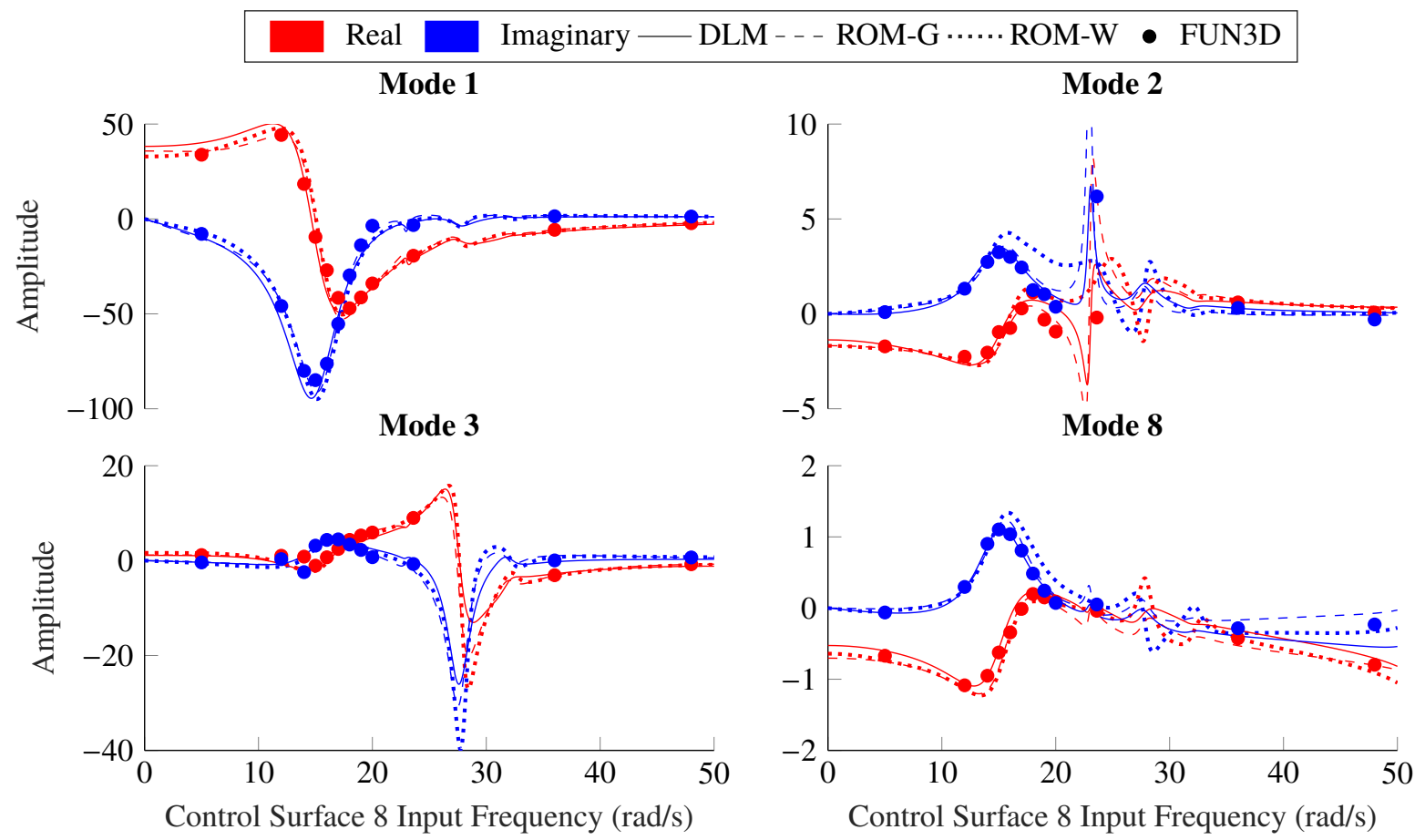

Fig. 5 Real and imaginary components of the modal displacements due to a sinusoidal modal displacement input to control surface \#2. $(q \approx 17 \mathrm{kPa})$ 
ROMs might have captured some nonlinearities that the DLM was unable to predict. For those frequency ranges where the ROMs did not clearly outperform the DLM, it might be that a Mach number of 0.85 and an angle of attack of $0^{\circ}$ (the flight conditions for these analyses) were not aggressive enough to induce the kinds of nonlinearities that might have been better captured by the ROMs.

While two ROMs were generated and were used in the previous comparison, only ROM-G was implemented into the ASE model. No controller was developed for ROM-W. As such, all subsequent results do not include ROM-W.

The closed-loop ASE model as shown in Fig. 4 was implemented and modal accelerations as predicted by FUN3D $(\ddot{\eta})$ were compared to the modal accelerations predicted by the linear codes $(\hat{x})$, which acted as the estimator in Fig. 4 Figure 6 shows these comparisons. It should be noted that the modal amplitudes are the only states that FUN3D and the linear aeroelastic models (DLM, ROM-G) had in common. The linear models were also defined by aerodynamic states (via the Roger approximation) that obviously did not exist in FUN3D. Also, note that, because these results come from the closed-loop ASE model, $\ddot{\eta}$ for each case was not identical since FUN3D was given commands from the controller that were based on $\hat{x}$, which varied between estimators. The results show that the linear codes predict the actual state well, but that, once again, the ROM does not clearly outperform the DLM.

\section{B. Effectiveness of Controllers}

Differences became more apparent when the effectiveness of each linear model's controllers were compared. Figure 7 shows unstable open-loop and stable closed-loop responses in modal displacement at a dynamic pressure of approximately $35 \mathrm{kPa}$. Note that the initial behavior in the response is a static aeroelastic simulation where no external disturbance had yet been introduced. The beginning of the dynamic aeroelastic simulation, where a disturbance in modal velocity for mode 1 was introduced, occurs just before 1 second. While both controllers were able to stabilize the system, the ROM-G-based controller clearly outperformed the DLM-based controller. The advantages of the ROM-G-based controller were further demonstrated as the dynamic pressure was increased. For each controller at each dynamic pressure, the response of mode 1 was fit to a curve of the form $\theta e^{\lambda t}$, where $\lambda$ is a measure of the decay of the system and is indicative of stability- positive $\lambda$ values indicate the system is unstable and negative values indicate the system is stable. Figure 8 shows the values of $\lambda$ for each controller as a function of dynamic pressure. The open-loop system

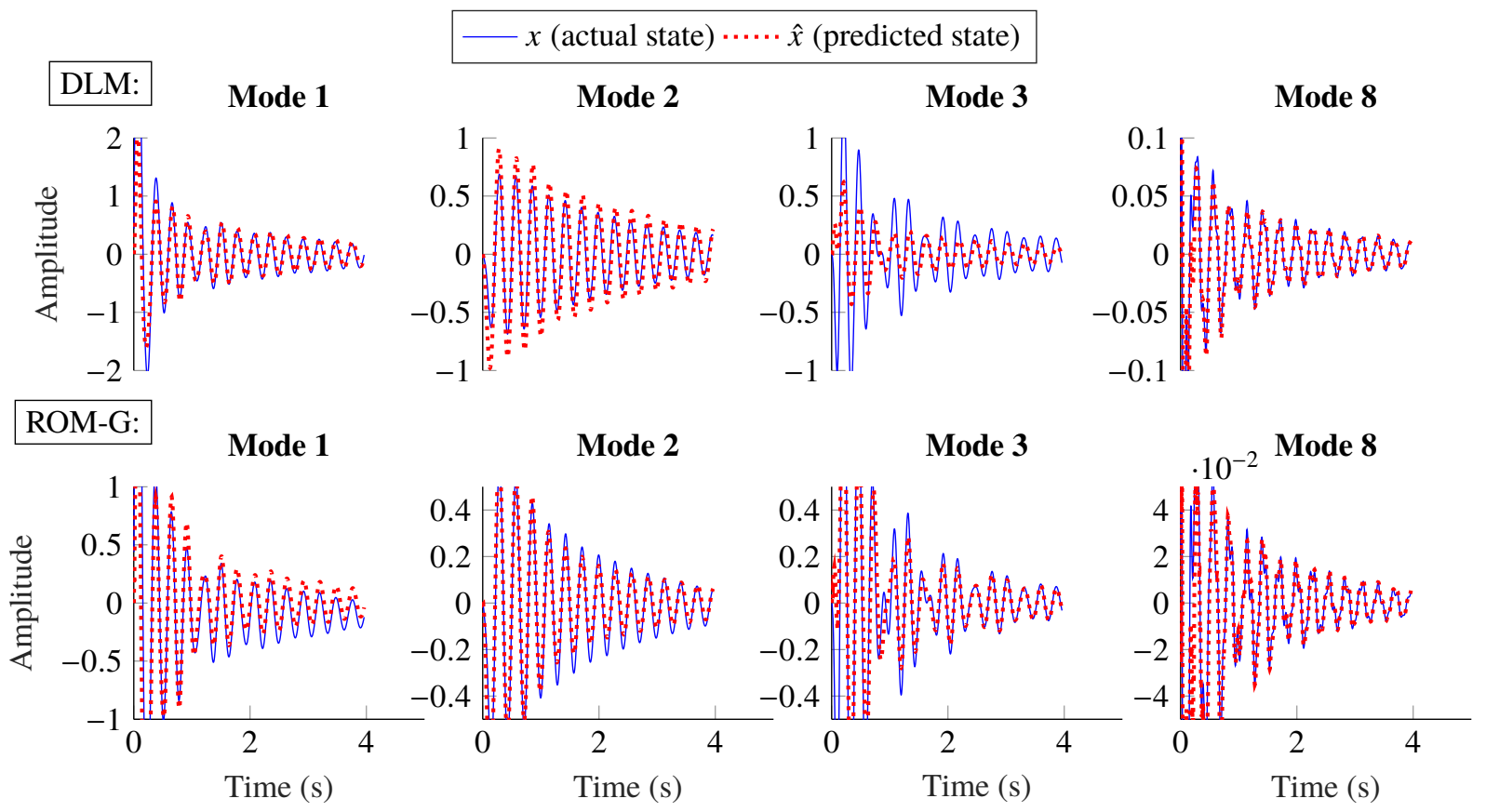

Fig. 6 Modal accelerations as predicted by FUN3D $(\ddot{\eta})$ compared to predicted modal accelerations $(\hat{x})$ by DLM (row 1) and ROM-G (row 2). Since the DLM is integrated in time alongside the closed-loop simulation in FUN3D, it is instructive to visualize how well the DLM is predicting what is actually happening in FUN3D. These plots show that the predictions are close to the actual states from FUN3D. 

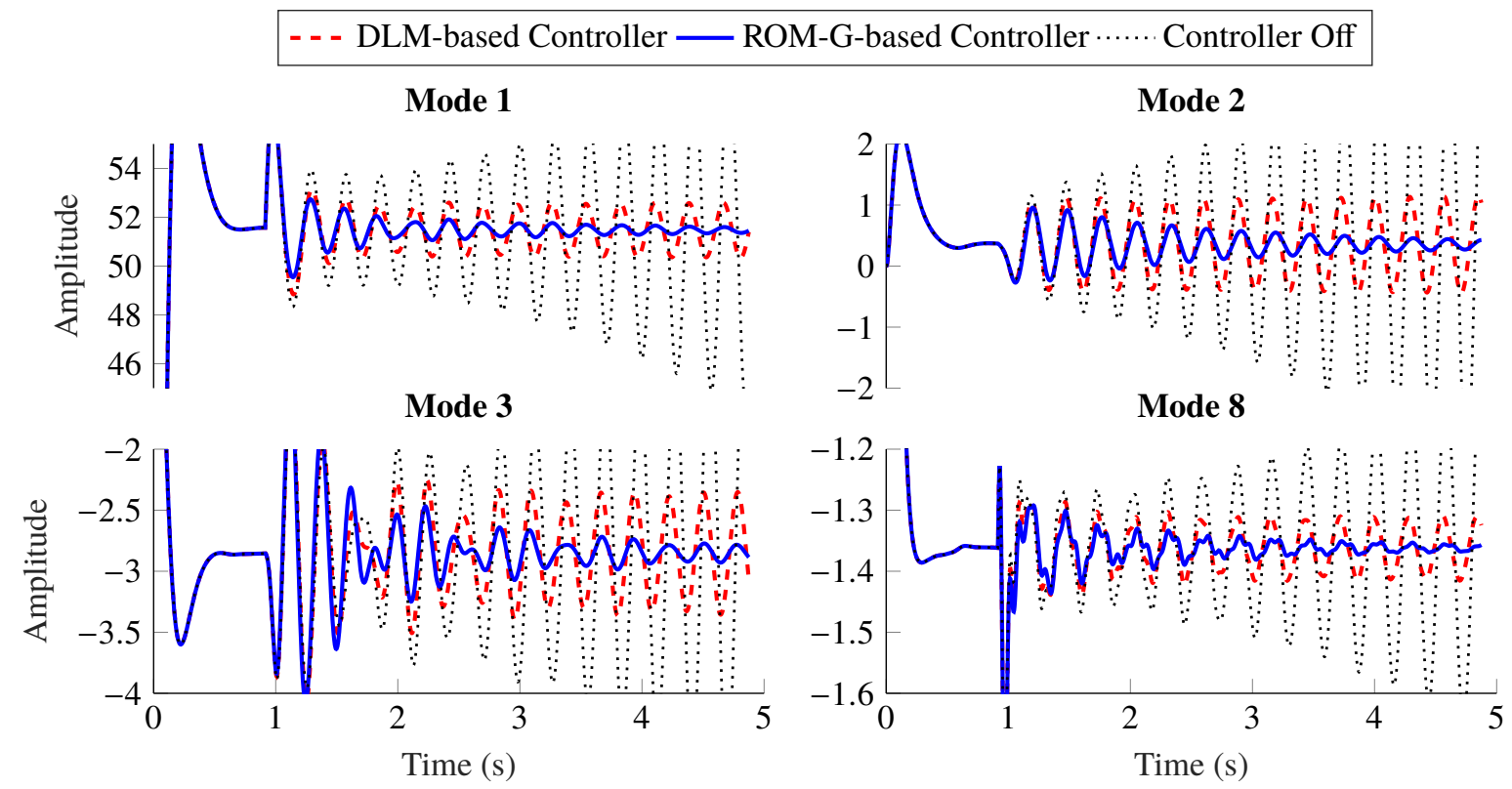

Fig. 7 The unstable open-loop response in modal displacement compared to a stabilized closed-loop response. For this simulation, a disturbance in modal velocity for mode 1 is introduced just before 1 second. $(q \approx 35 k P a)$

begins to go unstable at a dynamic pressure of about $30 \mathrm{kPa}$. The DLM-based controller is able to stabilize the system to higher dynamic pressures, but it too eventually goes unstable around a dynamic pressure of $35 \mathrm{kPa}$. The ROM-G-based controller stabilized the system for all tested dynamic pressures (up to approximately $60 \mathrm{kPa}$ ).

Even though there was not a clear advantage of using ROMs over the DLM to predict the aerodynamics given some control surface input (as shown in Fig. 5), significant differences in the closed-loop responses were seen between the controllers based on the DLM and the ROM. The ROM-based controller was significantly more successful at stabilizing

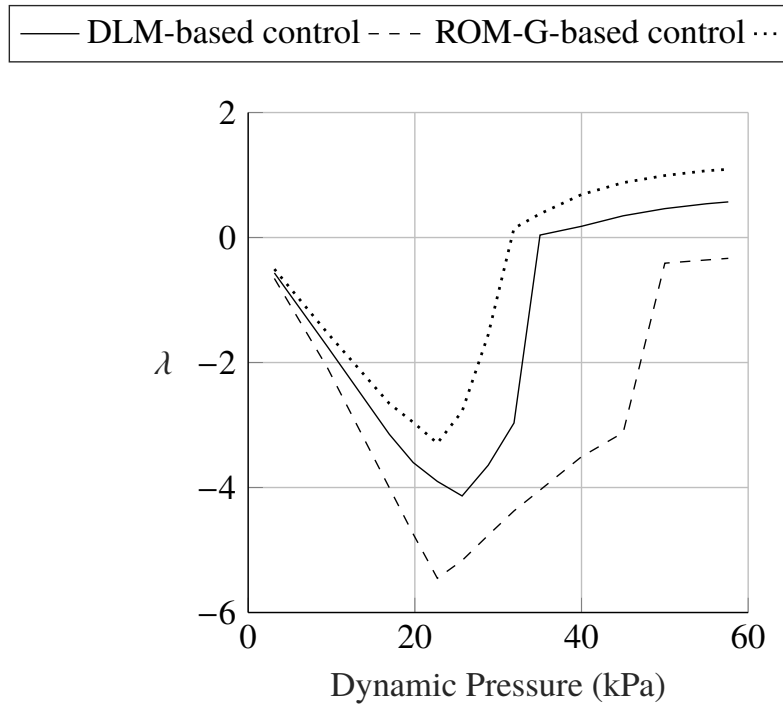

Fig. 8 A measure of the system's stability, $\lambda$, versus dynamic pressure. Positive $\lambda$ values imply that the system is unstable, while negative values indicate that the system is stable. The ROM-G-based controller does not go unstable for any dynamic pressures analyzed here, which demonstrates it improved ability over the DLM-based controller. 
the unstable system, presumably because of the ROM's ability to capture nonlinear aerodynamics that are beyond the DLM's ability to predict. It may be that the nonlinearities that are present at Mach 0.85 and at an angle of attack of $0^{\circ}$ are strong enough to see a difference in the closed-loop responses, but are not strong enough to see a difference in the predictions of aerodynamics between the ROM and the DLM. Future work, therefore, will include analyses of the system at higher Mach numbers or angles of attack where the nonlinearities should be stronger. In such conditions, the ROM should make better predictions of the aerodynamics than the DLM and should continue to outperform the DLM in controlling the system.

\section{Conclusion}

Active flutter suppression was successfully demonstrated within a computational ASE model that used a nonlinear aerodynamics solver (FUN3D) and a realistic airplane configuration. This was accomplished by using several linear methods to develop controllers. Results from several linear aerodynamics codes were compared to the nonlinear analyses, and two controllers were developed from the linear codes that were implemented inside the ASE model. The ROM-based controller stabilized the system better than the DLM-based controller and was effective over a larger range of dynamic pressures. It is believed that the ROM's ability to account for aerodynamic nonlinearities beyond what is captured by the DLM is the reason that the ROM-based controller was more successful than the DLM-based controller. Future work will include similar analyses at different flight conditions where nonlinearities in the flow might more strongly influence the solution.

\section{Acknowledgments}

This work is funded by the NASA Advanced Air Transport Technologies program. 


\section{References}

[1] Livne, E., "Aircraft Active Flutter Suppression: State of the Art and Technology Maturation Needs," Journal of Aircraft, 2017, pp. $1-41$.

[2] Mukhopadhyay, V., "Flutter Suppression Control Law Design and Testing for the Active Flexible Wing," Journal of Aircraft, Vol. 32, No. 1, 1995, pp. 45-51.

[3] Lu, Z., Cui, Y., Schneider, D., Zhao, Z., Chen, X., Lai, K. L., and Lum, K.-Y., "Aeroelastic Responses Identification of a High-Aspect-Ratio Flexible Wing Model and Its Active Flutter Suppression Strategy," 57th AIAA/ASCE/AHS/ASC Structures, Structural Dynamics, and Materials Conference, San Diego, CA, 4-8 Jan 2016.

[4] Qian, W., Huang, R., Hu, H., and Yonghui, Z., "Active Flutter Suppression of a Multiple-Actuated-Wing Wind Tunnel Model," Chinese Journal of Aeronautics, Vol. 27, No. 6, 2014, pp. 1451-1460.

[5] Block, J. J., and Strganac, T. W., "Applied Active Control for a Nonlinear Aeroelastic Structure," Journal of Guidance, Control, and Dynamics, Vol. 21, No. 6, 1998, pp. 838-845.

[6] Burnett, E., Beranek, J., Holm-Hansen, B., Atkinson, C., and Flick, P., "Design And Flight Test of Active Flutter Suppression on the X-56A Multi-Utility Technology Test-Bed Aircraft," The Aeronautical Journal, Vol. 120, No. 1228, 2016 , pp. 893-909.

[7] Ting, E., Chaparro, D., and Nguyen, N. T., "Development of an Integrated Nonlinear Aeroservoelastic Flight Dynamic Model of the Truss-Braced Wing Aircraft," 58th AIAA/ASCE/AHS/ASC Structures, Structural Dynamics, and Materials Conference, Grapevine, TX, 9-13 Jan 2017.

[8] Wang, Y., Wynn, A., and Palacios, R., "Nonlinear Modal Aeroservoelastic Analysis Framework for Flexible Aircraft," AIAA Journal, 2016, pp. 3075-3090.

[9] Zhao, Y., "Flutter Suppression of a High Aspect-Ratio Wing with Multiple Control Surfaces," Journal of Sound and Vibration, Vol. 324, No. 3-5, 2009, pp. 490-513.

[10] Biedron, R., Carlson, J.-R., Derlaga, J., Gnoffo, P., Hammond, D., Jones, W., Kleb, B., Lee-Rausch, E., Nielsen, E., Park, M., Rumsey, C., Thomas, J., and Wood, W., “FUN3D Manual: 13.1,” Tech. Rep. TM-2016-219580, NASA, 2017.

[11] Afonso, F., Vale, J., Oliveira, É., Lau, F., and Suleman, A., "A Review on Non-Linear Aeroelasticity of High Aspect-Ratio Wings," Progress in Aerospace Sciences, Vol. 89, 2017, pp. 40-57.

[12] Hallissy, B., and Cesnik, C., "High-Fidelity Aeroelastic Analysis of Very Flexible Aircraft," 52nd AIAA/ASME/ASCE/AHS/ASC Structures, Structural Dynamics and Materials Conference 19th AIAA/ASME/AHS Adaptive Structures Conference 13t, Denver, CO, 4-7 Apr 2011.

[13] Brooks, T. R., Kenway, G. K., and Martins, J., "Undeflected Common Research Model (uCRM): An Aerostructural Model for the Study of High Aspect Ratio Transport Aircraft Wings," 35th AIAA Applied Aerodynamics Conference, Denver, CO, 5-9 Jun 2017.

[14] Tantaroudas, N. D., and Da Ronch, A., "Nonlinear Reduced-order Aeroservoelastic Analysis of Very Flexible Aircraft," Advanced UAV Aerodynamics, Flight Stability and Control: Novel Concepts, Theory and Applications, 2017, p. 143.

[15] Danowsky, B. P., Thompson, P. M., Farhat, C., Lieu, T., Harris, C., and Lechniak, J., "Incorporation of Feedback Control into a High-Fidelity Aeroservoelastic Fighter Aircraft Model," Journal of Aircraft, Vol. 47, No. 4, 2010, pp. 1274-1282.

[16] Danowsky, B. P., Lieu, T., and Coderre-Chabot, A., "Control Oriented Aeroservoelastic Modeling of a Small Flexible Aircraft using Computational Fluid Dynamics and Computational Structural Dynamics-Invited," AIAA Atmospheric Flight Mechanics Conference, San Diego, CA, 4-8 Jan 2016.

[17] Selitrennik, E., Karpel, M., and Levy, Y., “Computational Aeroelastic Simulation of Rapidly Morphing Air Vehicles,” Journal of Aircraft, Vol. 49, No. 6, 2012, pp. 1675-1686.

[18] Kenway, G., Kennedy, G., and Martins, J., “Aerostructural Optimization of the Common Research Model Configuration,” 15th AIAA/ISSMO Multidisciplinary Analysis and Optimization Conference, Altanta, GA, 16-20 Jun 2014.

[19] Edwards, J. W., Bennett, R. M., Whitlow, W., and Seidel, D. A., “Time-marching transonic flutter solutions including angle-of-attack effects," Journal of Aircraft, Vol. 20, No. 11, 1983, pp. 899-906.

[20] Livne, E., “Integrated Aeroservoelastic Optimization: Status and Direction,” Journal of Aircraft, Vol. 36, No. 1, 1999, pp. $122-145$. 
[21] Roger, K., “Airplane Math Modeling Methods for Active Control Design,” AGARD CP-288, 1977.

[22] Bartels, R. E., "Flexible Launch Stability Analysis Using Steady and Unsteady Computational Fluid Dynamics," Journal of Spacecraft and Rockets, Vol. 49, No. 4, 2012, pp. 644-650.

[23] Bartels, R. E., and Stanford, B., "Economical Unsteady High Fidelity Aerodynamics in a Structural Optimization with a Flutter Constraint," 35th AIAA Applied Aerodynamics Conference, Denver, CO, 5-9 Jun 2017.

[24] Silva, W. A., "AEROM: NASA's Unsteady Aerodynamic and Aeroelastic Reduced-Order Modeling Software," Aerospace, Vol. 5, No. 2, 2018.

[25] Bhoir, N., and Singh, S. N., "Output Feedback Nonlinear Control of an Aeroelastic System with Unsteady Aerodynamics," Aerospace Science and Technology, Vol. 8, No. 3, 2004, pp. 195-205.

[26] Librescu, L., and Marzocca, P., "Advances in the Linear/Nonlinear Control of Aeroelastic Structural Systems," Acta Mechanica, Vol. 178, No. 3-4, 2005, pp. 147-186.

[27] Stevens, B. L., Lewis, F. L., and Johnson, E. N., Aircraft Control and Simulation: Dynamics, Controls Design, and Autonomous Systems, John Wiley and Sons, 2015. 